\title{
Corona discharge pulse pattern and audible noise on hybrid AC/ DC transmission lines under electric field bias, ripple and ion coupling
}

\author{
Journal Article \\ Author(s): \\ Hedtke, Sören (1); Pfeiffer, Martin D.; Franck, Christian (D) \\ Publication date: \\ 2019-11 \\ Permanent link: \\ https://doi.org/10.3929/ethz-b-000368955 \\ Rights / license: \\ Creative Commons Attribution-NonCommercial-NoDerivatives 4.0 International \\ Originally published in: \\ Journal of Electrostatics 102, https://doi.org/10.1016/j.elstat.2019.103373
}




\title{
Corona Discharge Pulse Pattern and Audible Noise on Hybrid AC/DC Transmission Lines under Electric Field Bias, Ripple and Ion Coupling
}

\author{
Sören Hedtke ${ }^{\mathrm{a}}$, Martin Pfeiffer ${ }^{\mathrm{a}}$, Christian M. Franck ${ }^{\mathrm{a}}$ \\ ${ }^{a}$ High Voltage Laboratory, ETH Zurich, Physikstrasse 3, CH-8092 Zurich, Switzerland
}

\begin{abstract}
Hybrid AC/DC overhead lines offer a significant increase in transmission capacity compared to existing HVAC infrastructure. While corona phenomena and audible noise are crucially important for public acceptance, they are affected by mutual AC/DC coupling. While the underlying phenomena need to be clearly understood and predicted accurately, only few experimental studies exist. The corona effects are therefore studied in detail on a large laboratory setup under the influence of electric field bias, ripple and space charges. The results show a clear trend in the phase resolved partial discharge (PRPD) pattern and repetition rate which can explain the change in audible noise.
\end{abstract}

Keywords: corona effects; audible noise; partial discharge; hybrid tower; AC/DC transmission line; corona pulse characteristics

\section{Introduction}

Many utilities are faced with the challenge of large required grid reinforcements to cope with the rapid installation of renewable energy sources (RES). The concept of 5 hybrid $\mathrm{AC} / \mathrm{DC}$ lines is motivated by the possibility of increased power transmission capacity using existing tower geometries. Under the assumption of higher acceptance due to negligible visual change, a faster grid reinforcement can be achieved [1]. This offers a clear advantage in contrast to long regulatory procedures for new overhead line corridors or the high financial and technical effort of underground cable or gas-insulated lines. Hybrid AC/DC overhead lines benefit from the increased transmission capacity of the DC conversion due to higher voltages and lower ac15 tive and reactive losses. At the same time, the remaining AC system allows to keep important nodes in populated areas and continues supporting the HVAC grid. Therefore, hybrid AC/DC lines are currently discussed in various countries [2], e.g. Germany [3, China [4, the United 20 States and Switzerland [5]. While the visual impact of a hybrid conversion is assumed to be negligible, environmental effects, such as audible noise and electromagnetic fields caused by corona discharges will be altered.

If the electric field on the conductor surfaces exceeds the through water drops, pollution or scratches, a corona discharge occurs [6]. This corona current pulse manifests itself as an electromagnetic interference [7], as well as an acoustic pulse through the local heating of the air. Due to

\footnotetext{
*Corresponding author: Christian M. Franck

Email address: cfranck@ethz.ch (Christian M. Franck)
}

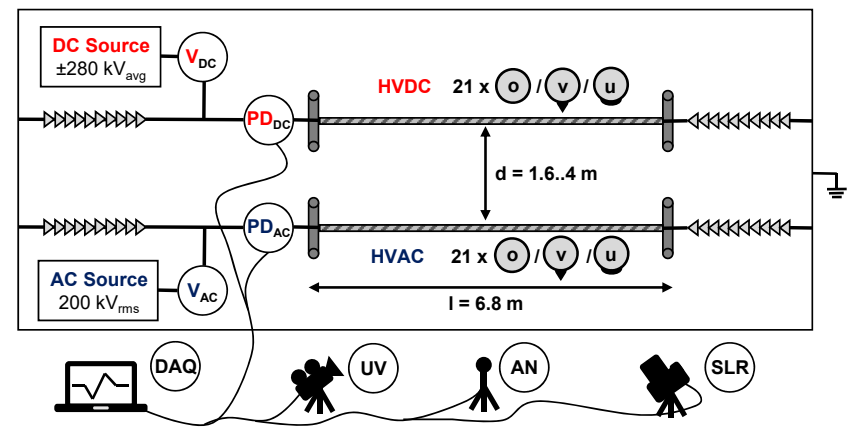

Figure 1: Indoor hybrid AC/DC test using two parallel conductors with either round (u), spiky (v) or no artificial corona sources (o)

their random individual placement and occurrence, this causes radio interference (RI) and audible noise (AN), which can be perceived as a nuisance. Furthermore, an ion cloud will be generated in the electric field. Ions will move periodically in the conductor vicinity in alternating fields, causing the well-known hum component from HVAC overhead lines [8], while in HVDC these ions are repelled from the conductor of the same polarity and are attracted by the ground as well as adjacent conductors of different potentials. This steady DC ion drift causes a measurable DC ion current density and an increased Poisson electric field at ground level 6]. Thus, the hum component vanishes for HVDC overhead lines, as no periodic ion movement occurs [9]. Furthermore, the ion cloud is considered to be the cause for the drop in HVDC radio and audible noise for a high density of corona sources, e.g. during wet weather conditions 9, 10. Experience from outdoor tests 


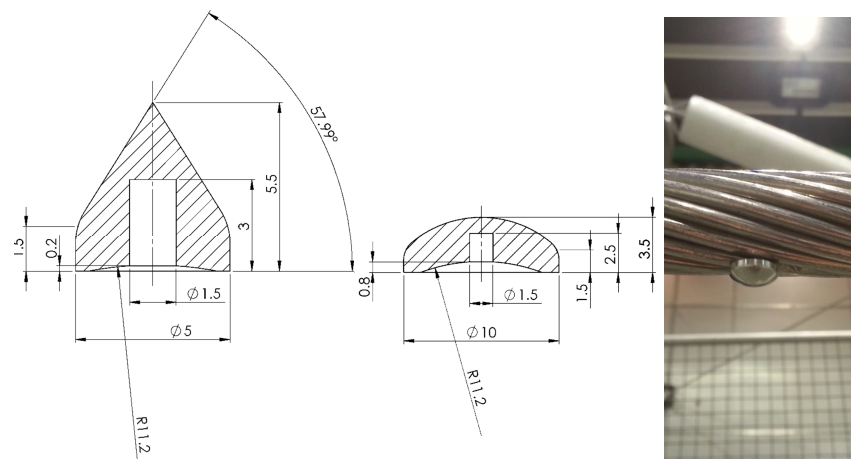

Figure 2: Photo of round u-type source on conductor and geometry sketch for $\mathrm{v}$-type and $\mathrm{u}$-type artificial sources (in $\mathrm{mm}$ )

has shown that HVDC radio and audible noise is predominantly caused by the positive pole due to its attraction of 105 the highest levels of HVDC noise can be expected from the positive pole in summer while the HVAC noise is caused by all phases and is typically worst for high precipitation [12, 8.

55 The concept of hybrid AC/DC lines as well as its corona characteristics were first studied in the US and Canada by BPA [13, EPRI [14] and IREQ [15]. Due to the presence of both AC and DC voltages, significant radio and audible noise levels can occur during both dry and wet115 60 weather. Additionally, the low separation distance between AC and DC facilitates interferences between both systems. Through electrostatic induction the AC surface gradient will experience a DC bias caused by adjacent DC bundles. Likewise, the $\mathrm{AC}$ bundles will enforce a ripple on ${ }^{120}$ 65 the DC surface gradient of the HVDC poles [13]. Furthermore, the DC bundles are subject to an AC current due to capacitive and inductive coupling [7]. Also, the ion drift will cause a DC ion current coupling between the DC and AC conductors [16]. Similar to the enhanced Poisson elec-125 70 tric field at ground due to the additional space charges, also the surface gradient can be expected to be affected in addition to the bias without any ions based on simulations by Maruvada and Drogi [15. As audible noise is a major criterion for the acceptance of new and converted overhead lines alike [1, a clear understanding of the underlying phenomena as well as its accurate prediction are of 130 high interest. However, only very few experimental studies have been carried out so far, with somewhat inconclusive results, especially with regard to the DC bias and its sever-

80 ity on the audible noise [13, 14, 17] as well as the effect of a $\mathrm{DC}$ ripple on the $\mathrm{AC}$ corona noise.

This study therefore aims to investigate the corona characteristics of hybrid lines in an indoor setup using a parallel arrangement of two conductors energized with $\mathrm{AC}$ and DC respectively. A previous publication by the authors has shown that the corona audible noise increases when 140 $\mathrm{AC}$ corona is subject to a positive bias, as well as when $\mathrm{DC}$ corona is affected by an AC ripple [17]. However, the A-level audible noise is only a macroscopic character0 istic which blurs out the differences between the different corona sources and their activities. Furthermore, the audible noise in many studies is subject to background noise due to weather, voltage sources and other distortions. This study therefore performs an additional partial discharge 95 measurement in order to explain the observed behavior based on the detailed discharge characteristics. Additionally, near-UV long-exposure photography will be used to observe the number and activity of corona sources for every voltage step from the exact same angle. This allows to verify that indeed the conductor is free of corona or actually shows much brighter and stronger Streamer formation. Also, the effect of a negative bias on the audible noise will be addressed which is still unclear based on the previous investigations.

As shown by [18, while using metallic spheres as corona sources, there is a strong correlation in amplitude of the corona current pulses and the acoustic pulses for positive DC corona as well as for AC corona [19. Likewise, it was demonstrated by EPRI that the trends of audible noise and radio interference voltage show a very strong correlation [14. A study from the North China Electric Power University (NCEPU) has shown that an AC ripple causes a change in the DC corona current pulse patterns in a corona cage experiment. Based on these results, the corona pulse patterns are of great interest for the case of parallel corona on two adjacent AC and DC conductors. Based on the theoretical discussion by 8 , the acoustic energy is higher for one single corona pulse than for two pulses of half its energy due to the energetic addition. Therefore, in the authors opinion, the corona audible noise is dominated by the high amplitude pulses. Based on the studies of Trinh [20], the pulses of the positive streamer discharges are typically higher and of lower repetition rate than the negative Trichel discharges, which can partially explain the lower audible noise for negative DC corona. Therefore this study will focus on the corona pulses under mutual $\mathrm{AC} / \mathrm{DC}$ coupling, especially regarding the amplitude and frequency of strong positive pulses.

\section{Experimental Setup and testing procedure}

The power supply is realized by cascading MWB high voltage building elements. The applicable maxima are $200 \mathrm{kV}$ for $\mathrm{AC}$ (rms) and $240 \mathrm{kV}$ for DC (average). The voltage supply is controlled using a frequency converter as presented in [21] and can be regulated remotely using MATLAB. For the hybrid test line, two untreated ACSR 264-AL1/34-ST1A conductors with a diameter of $22.4 \mathrm{~mm}$ are strung up in the air as shown in Figure 1.

The maximum surface gradients of the $\mathrm{AC}$ and $\mathrm{DC}$ conductor are derived from a 2D COMSOL simulation at maximum sag based on the assumption of round and infinitely long conductors, which is typical for the field of corona and audible noise [6, 12. As the setup is symmetrical, the ratio of surface gradient over applied voltage is identical 


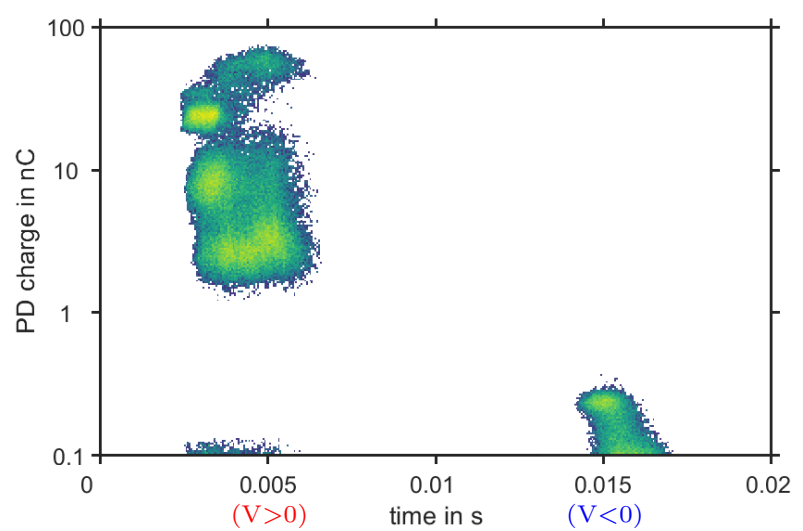

(a) Corona PRPD (DC $=-240 \mathrm{kV})$

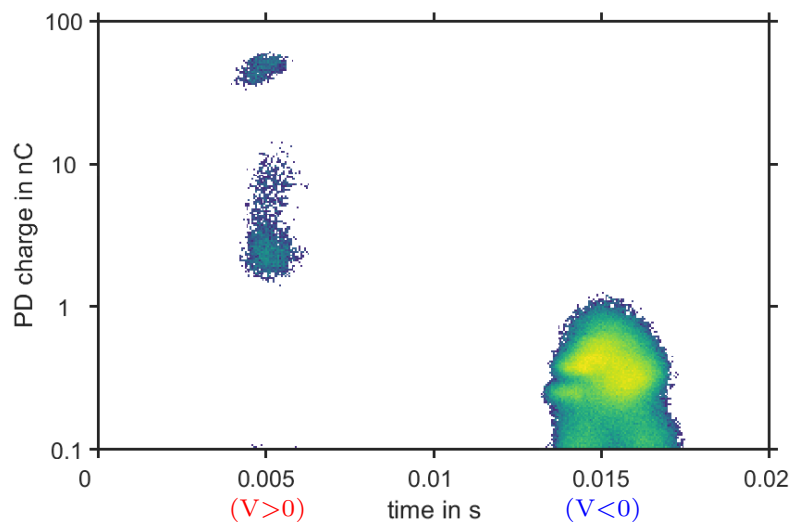

(c) Corona PRPD $(\mathrm{DC}=0 \mathrm{kV})$

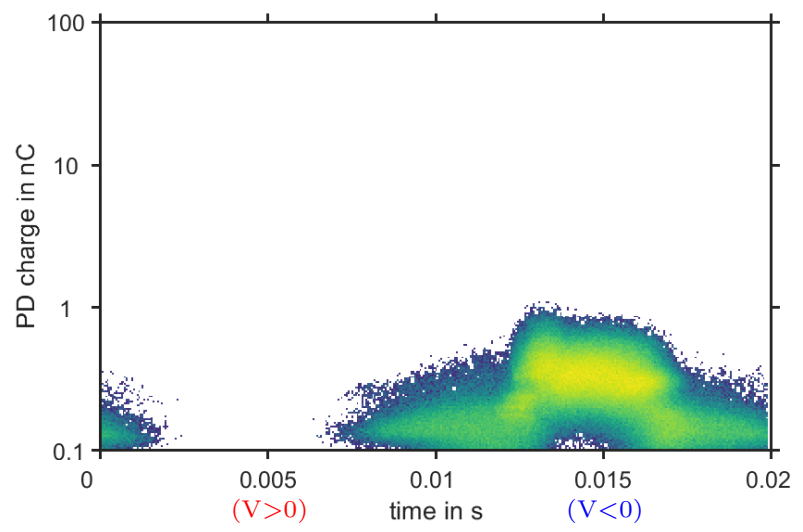

(e) Corona PRPD $(\mathrm{DC}=240 \mathrm{kV})$

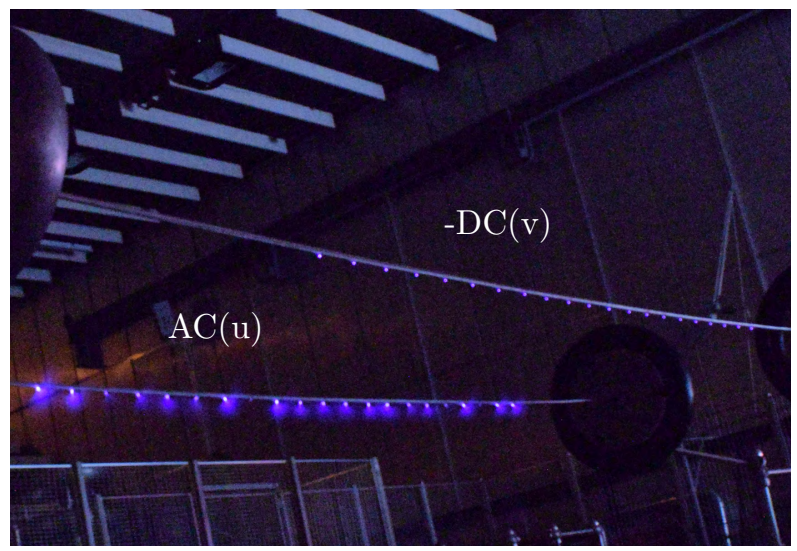

(b) Corona activity $(\mathrm{DC}=-240 \mathrm{kV})$

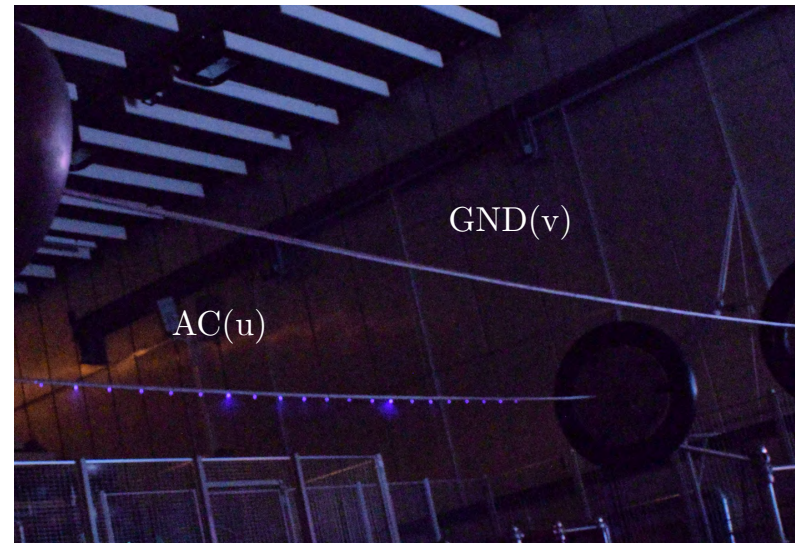

(d) Corona activity $(\mathrm{DC}=0 \mathrm{kV})$

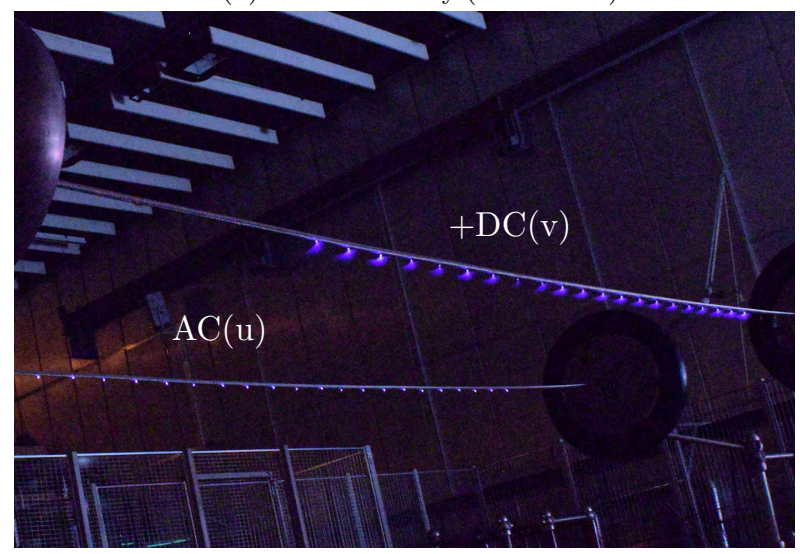

(f) Corona activity $(\mathrm{DC}=240 \mathrm{kV})$

Figure 3: Comparison of AC corona PRPD and UV activity at $180 \mathrm{kV}$ for positive, no and negative bias for $240 \mathrm{kV}$ DC

at $16.2 \mathrm{kV} / \mathrm{cm}$ per $100 \mathrm{kV}$ for both systems. The coupling ${ }_{155}$ 145 surface gradient of $2.3 \mathrm{kV} / \mathrm{cm}$ per $100 \mathrm{kV}$ on the adjacent grounded conductor and then added based on the superposition principle. The relative bias and ripple ratios are calculated based on the electric field amplitudes instead of ${ }_{160}$ To control the state of corona on both conductors individually, 21 artificial corona sources made of aluminum are placed equally spaced on the conductor surface. These artificial corona sources show similar results to real rain ${ }_{165}$

drops, as demonstrated by [10, while being very stable and easy to control. Additionally, this also enables to change corona pulse parameters and its repetition rate using different shapes as shown by [22. Using this concept, corona sources can be designed to either generate a large ion current without significant audible noise, further-on referred to as v-shape (v), or vice versa generate high audible noise with small corona losses, the so-called u-shape (u), as demonstrated in [17] and shown in Figure 2 This design has shown to generate reproducible results and was also implemented in [16. Also, in order to allow electro- 
static coupling without ion current coupling, the conductor causing the coupling was not equipped with (v) or $(\mathrm{u})$ type sources, but was kept clean, dry and free of corona sources and will be referred to as (o) case.

170 For each configuration, the voltage was ramped up automatically on one side with a total of 121 steps while the other side was kept at constant voltage. For each step, the voltage was kept constant for 60 seconds. During every step, a long exposure photograph of $30 \mathrm{~s}$ was taken with ${ }_{230}$ a Nikon D5300 digital single-lens reflex (DSLR) camera to monitor the state of corona and the number of active sources, as shown for example in Figure 3f The camera was positioned at the edge of the safety cage with $5.9 \mathrm{~m}$ distance to the mid span in order to be as close as possi-235 ble while still allowing to capture both conductors at the same time. Additionally, a corona camera was used from different angles to ensure that no PD occurs on the high voltage electrodes and connectors. The corona pulses are measured using the partial discharge measurement system 240 Omicron MPD 600 with a measuring impedance in series with the coupling capacitor. The setup was calibrated using a pulse generator according to the IEC 60270 standard. The center frequency was chosen at $250 \mathrm{kHz}$ within a range of $+-150 \mathrm{kHz}$. The device was set to auto-range, as otherwise saturation effects were unavoidable due to the large dynamics of the input signal at different voltage levels. The applied voltage was measured using calibrated ${ }^{245}$ $\mathrm{AC}$ and DC voltage dividers and recorded with a LabJack U3-HV A/D converter. To measure the audible noise of the conductors, two Norsonic Nor118 sound level meters with Class-1 free field microphones were placed on both sides behind the cage with $2.1 \mathrm{~m}$ distance to the $\mathrm{AC}$ and ${ }^{250}$ DC conductors, allowing to capture the A-weighted Sound pressure levels as well as the third-octave frequency bands.

\subsection{Evaluation procedures}

For every voltage step, a stream of $30 \mathrm{~s}$ is extracted ${ }^{25}$ from the MPD 600 recording, including the apparent charge and time stamp of every detected discharge. The raw data is then filtered with Matlab. From this stream, all discharges smaller than $0.1 \mathrm{nC}$ are discarded, as their contribution to the audible noise is assumed very small com- ${ }^{26}$ pared to the other discharges of up to some ten $\mathrm{nC}$. While the phase relation is not available as raw data, it is reconstructed based on the time stamps with the assumption that the maximum discharge detected is always positive and occurs at the maximum of the positive half-wave. ${ }^{26}$ Hence, the positive amplitude correlates with $5 \mathrm{~ms}$ or 90 degrees. The phase relation of all other discharges is then reconstructed based on their time stamp. For a separated evaluation of the pulses in the positive and negative halfwave, it is assumed that all pulses before 180 degrees or ${ }^{27}$ $10 \mathrm{~ms}$ respectively belong to the positive half-wave and all after $10 \mathrm{~ms}$ are part of the negative half-wave. For both half-waves, the pulse amplitude percentiles are evaluated, as well as the number of pulses per second as a representation of the repetition rate. For every individual voltage step, a heat map is generated based on the phase shift and weighted by the number of pulses as in typical representations of $\mathrm{PD}$ patterns. To evaluate the trend of the repetition rate, the number of positive pulses per seconds is measured, e.g. in Figure 4b, Regarding the trend in pulse amplitude, the 99.9th percentile of the positive pulses is evaluated in order to track the behavior of the maximum pulse amplitudes as in Figure 4a.

Long-exposure photography highlighting the number of active corona sources and a qualitative evaluation of their activity, is used to detect the corona onset on both sides. For higher coupling ratios in case (o), corona onset was detected on the conductor although no artificial sources were attached. These voltage steps were consequently excluded in the post-processing, as they do not fulfill the assumption of no corona. This can occur, as the corona onset was tested prior to the measurements based on the energization of only the AC or DC system and can shift based on the hybrid energization as demonstrated in a previous publication of the authors [17.

\section{AC discharge behavior under positive and neg- ative DC bias}

\subsection{Measurement results}

The AC corona characteristics are investigated regarding the energization of an adjacent DC conductor with negative and positive polarity, respectively, inducing positive and negative DC bias. Furthermore, based on previous investigations [17, a distinction is made between DC energization with and without corona activity and hence ion current coupling. Consequently, the DC side was equipped with 21 artificial v-shape sources (v) for strong ion current coupling, while the conductor was clean, dry and free of corona for the cases without space charge effects (o). Exemplarily for the qualitative change in the PD pattern relative to phase, the PRPD patterns are shown for the two cases of strong coupling $\left(240 \mathrm{kV}_{\mathrm{DC}}\right.$ or a relative bias of $21.6 \%$ based on the AC amplitude) for positive (Figure 3a) and negative polarity (Figure 3e compared to a grounded DC conductor (Figure 3c). While the AC corona behavior looks almost identical for both cases without bias, as expected, the number of pulses increases in the positive half-wave while decreasing in the negative half-wave for the case of positive bias (Figure $3 a$ and vice versa for the negative bias (Figure 3e). Also, the range of phase angles for which positive and negative pulses occur is enlarged or reduced for the respective bias.

As a quantitative manifestation of these phenomena, the pulse amplitudes and repetition rates responsible for the audible noise [20] are shown for all gradients and test cases over the relative coupling based on the PD apparent charge and number of detected pulses. For the case of positive bias in Figure 4a the pulse amplitudes in the positive halfwave are increased. This is also true for the repetition rate in Figure $4 \mathrm{~b}$ The opposite could also be confirmed for the 


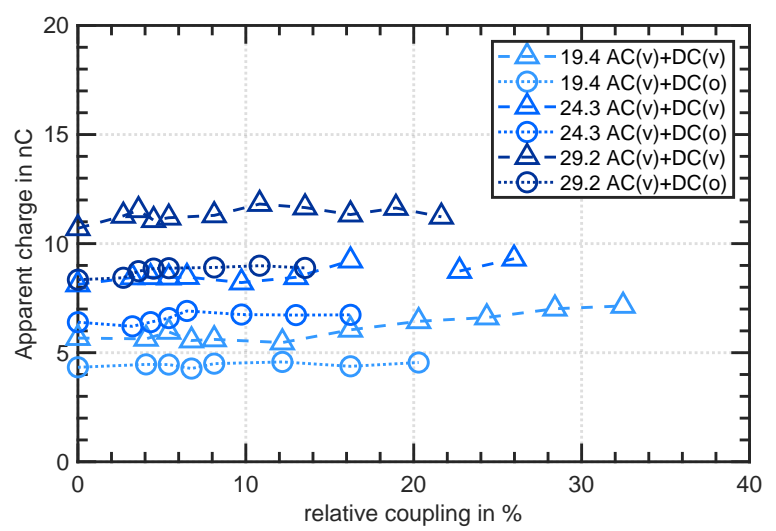

(a) Max. pos. amplitude - pos. bias

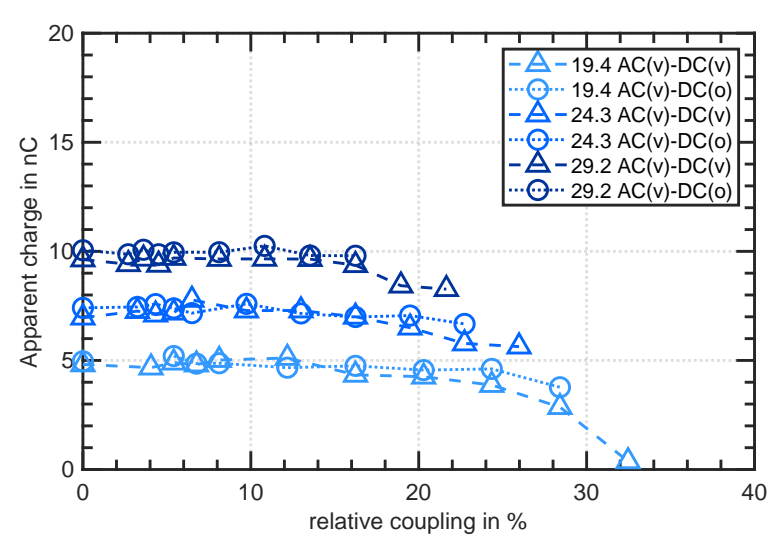

(c) Max. pos. amplitude - neg. bias

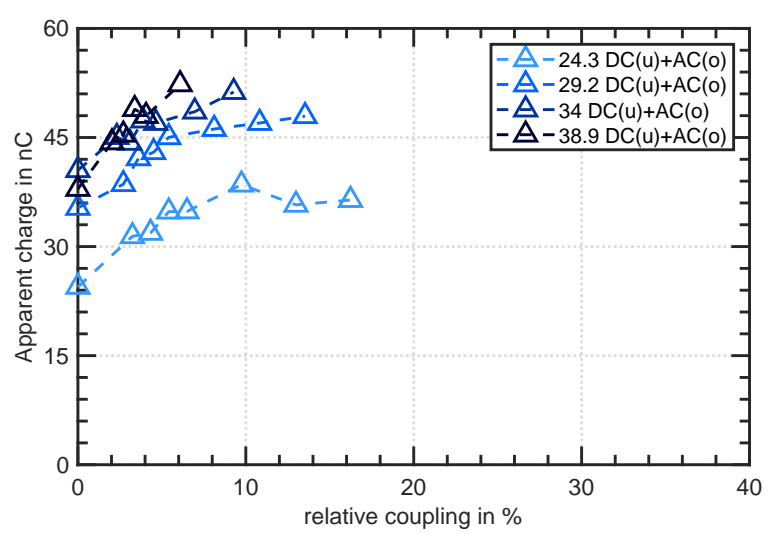

(e) Max. pos. amplitude - AC ripple

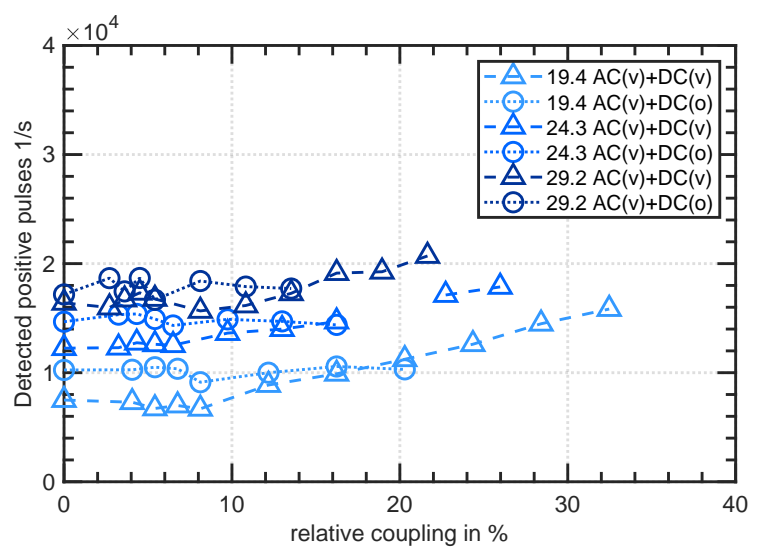

(b) Pos. repetition rate - pos. bias

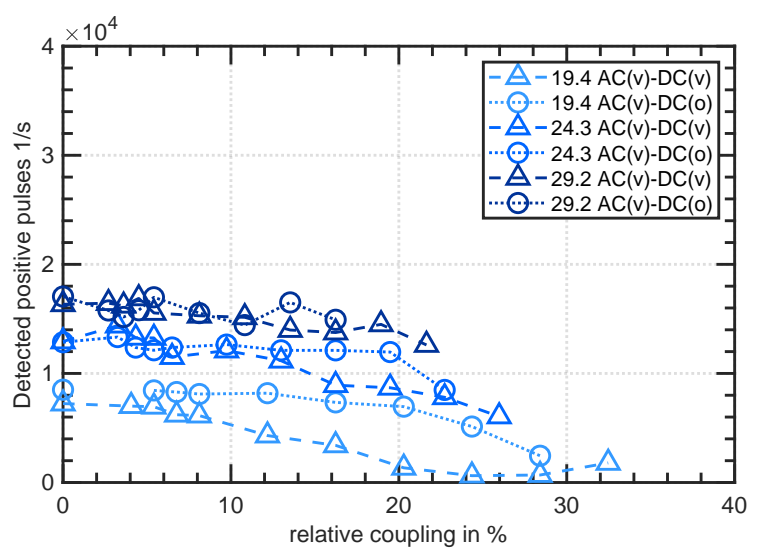

(d) Pos. repetition rate - neg. bias

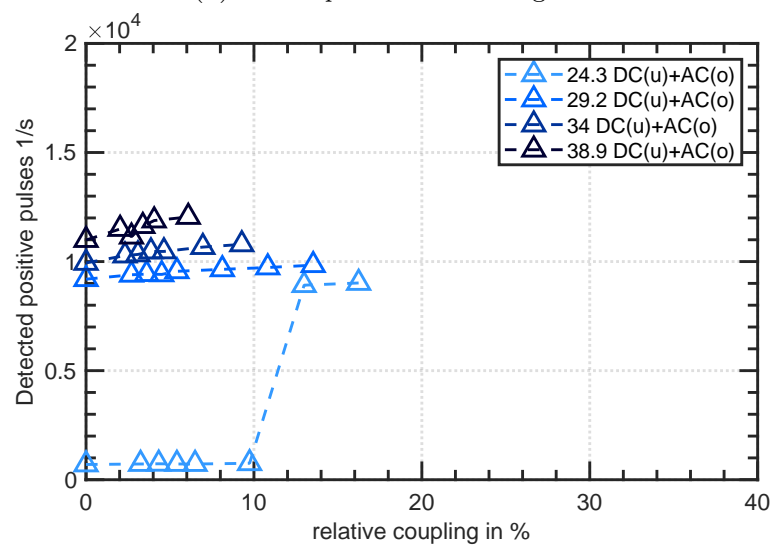

(f) Pos. repetition rate - $\mathrm{AC}$ ripple

Figure 4: Positive corona pulse characteristics for varying relative coupling (in \%) and applied AC field $(\mathrm{kV} / \mathrm{cm})$

negative half-wave, where both amplitude and frequency are reduced. In the case of negative bias in Figure $4 \mathrm{~d}$ this behavior is reversed. The positive pulse amplitudes are clearly reduced with the relative bias Figure $4 \mathrm{c}$ as well as for all AC surface gradients, the effect of relative coupling is found to be stronger for the lower AC gradients. In extreme cases, as for the lowest $\mathrm{AC}$ gradient, the negative coupling can even cause the positive discharges to extin- tatively for the two cases of positive and negative bias in Figure $3 \mathrm{~b}$ and Figure $3 \mathrm{f}$ It is obvious that no corona activity is observed on the DC conductor for the case of zero DC voltage. The energization of the $\mathrm{DC}$ conductor is visible for both cases, and the smaller negative Trichel discharges can well be distinguished from the positive Streamer discharges. Regarding the AC corona activity, all artificial corona sources are active in all cases for the selected $\mathrm{AC}$ gradients. While the image quality does not allow for a quantitative evaluation, the photographs still allow for a 
qualitative comparison of the AC corona activity with and without bias. Regarding the positive bias (Figure 3b), the corona activity is brighter compared to the case of no DC corona (Figure 3d). The AC discharges appear to be 355 300 longer and wider with the DC bias. On the contrary, the AC corona including negative DC bias (Figure $3 \mathrm{f}$ ) clearly reduces the brightness and footprint of the $\mathrm{AC}$ discharges. As the measurement was subject to high background audible noise from the frequency converter, the corona audible 360 noise is only presented for the highest voltage level to ensure a high signal to noise ratio. As a comparison of the trends of audible noise and corona pulses, the change in apparent $\mathrm{PD}$ charge $Q_{\max }$, as well as in the A-level AN is demonstrated for the case of bias in Figure 5 For the 365 positive bias, both the Audible Noise and the PD Amplitude increase. For the case of a strong negative bias, not only the PD characteristics decreased, but also the audible noise.

\subsection{Discussion}

The partial discharge characteristics and the audible noise are altered due to the coupling between AC and DC. All corona effects are closely connected to the surface gradient and will increase if also the maximum surface gradi-375 ent is increased. Due to the energization of adjacent AC and DC bundles, the surface gradient is enhanced based on the superposition of various coupling phenomena which are discussed based on the model in Figure 6. Regarding AC corona, the DC energization will cause a DC bias through ${ }_{380}$ electrostatic induction shown in Figure 6(d). The DC bias causes a shift in the surface gradient on the conductor depending on the DC polarity. For one half-wave, the maximum field amplitude is increased and so is the time period for which the critical field strength is exceeded. There- 385 fore, it is logical, that a positive bias causes an increased number and amplitude of positive pulses, while for negative pulses the phase range in which they occur as well as their amplitude are reduced. It can be assumed that the streamer integral is larger for pulses of one half-wave and ${ }_{390}$ that longer streamers can form, causing higher charge per pulse, and hence higher thermal and acoustic energy.

In case of ion current coupling between both $\mathrm{AC}$ and $\mathrm{DC}$, as shown in the case with v-type sources on both, the $\mathrm{AC}$ and DC conductor, multiple mechanisms exist, which will occur simultaneously and cannot generally be treated independently. If only the $\mathrm{DC}$ corona causes corona, e.g. due to increased pollution on the DC side, the DC ions will be repelled by the $\mathrm{DC}$ conductor and drift to the $\mathrm{AC}$ conductor with a superimposed oscillation caused by the $\mathrm{AC}$ field shown in Figure 6(a). This can be measured as a DC in the $\mathrm{AC}$ conductor. Furthermore, the DC ions drifting to the $\mathrm{AC}$ conductor will cause a locally enhanced ${ }^{400}$ space charge density in the conductor vicinity. The drift of negative ions to the $\mathrm{AC}$ conductor should further enhance a positive bias on the AC surface gradient. Likewise, it 350 is logical that positive ions will reduce the electric field in the positive $\mathrm{AC}$ halfwave, thereby supporting a negative bias.

If only the $\mathrm{AC}$ conductor would be in corona, e.g. due to different conductor types or scratches on the conductor, still a DC ion current would flow, as the AC ions are attracted by the steady DC field (Figure 6(c)) as shown previously in [16]. This removal of space charge from the $\mathrm{AC}$ conductor should further enhance the effect of the DC bias, as the removal of positive ions will enhance the surface gradient in the positive halfwave, as the shielding effect is reduced. The same is true for the removal of negative ions for a positive DC polarity.

However, in most cases, precipitation as the main source of corona will cause discharges to occur on both, AC and DC, simultaneously. In this case, the bipolar ion flow will show different mechanisms, e.g. due to recombination of ions (Figure 6(b)), which has shown to further increase the corona current coupling according to [16] in the socalled 'Hybrid Effect'. Here the DC ion current in the AC bundle was observed much stronger than the superposition of only $\mathrm{AC}$ or $\mathrm{DC}$ corona with the consequence of higher DC current on the $\mathrm{AC}$ side and a higher density of space charges. For the example of a positive bias from a negative $\mathrm{DC}$ conductor, the negative ions close to the $\mathrm{AC}$ conductors are assumed to further increase the bias in the same direction as the purely electrostatic coupling without ion current coupling. As shown previously by Maruvada and Drogi [23], corona from the DC side can cause the bias to further increase by more than $200 \%$ depending on the tower configuration. Therefore it is obvious, that the hybrid effect of simultaneous AC and DC corona should always force a change in the corona effects in the same direction as the electrostatic induction without space charge. This effect of the space charges was also shown to increase the audible noise in 17. Based on Figure 4, it can also be seen, that the pulse amplitudes and repetition rates are more affected for the case of DC ion current coupling. In some cases, the hybrid energization caused a dry and clean conductor to cause corona as well, which could be verified with the UV and PD data. As in this case, the assumption of purely electrostatic coupling without ion current coupling would no longer be valid these points were excluded accordingly.

\section{DC discharge behavior under an AC ripple}

\subsection{Measurement results}

Based on the previously observed effect of an AC ripple on the DC audible noise in [17, the DC corona current characteristics are now studied in detail regarding an energization of an adjacent AC conductor. As DC corona noise is typically worst in dry weather due to pollution [9], it is assumed that in this case only the DC conductor is coronating, while the AC conductor only causes an electrostatic coupling. As an example for this case, the corona PRPDs are shown for the two cases of no ripple (Figure 7c and a strong ripple of $19.5 \%$ in (Figure $7 \mathrm{~d}$ ). 


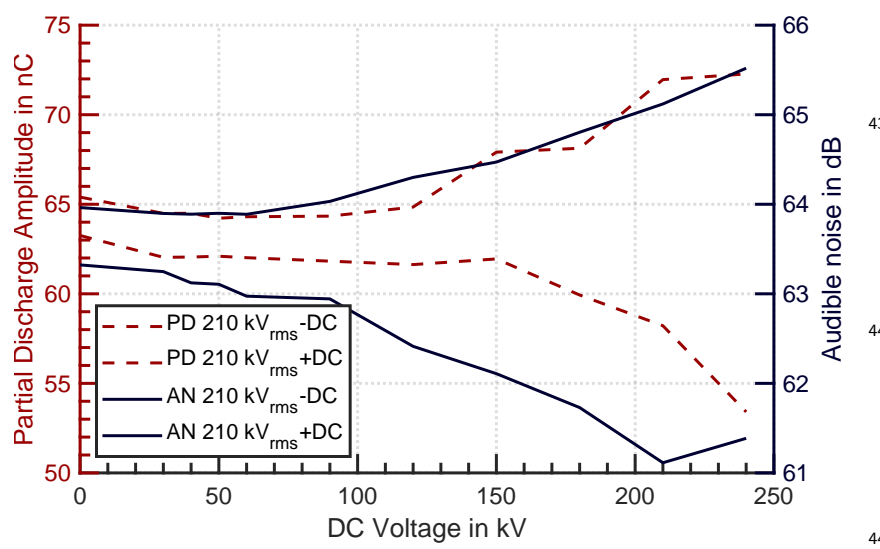

Figure 5: $\mathrm{PD}$ and $\mathrm{AN}$ characteristics for positive and negative bias

(a) Repulsion

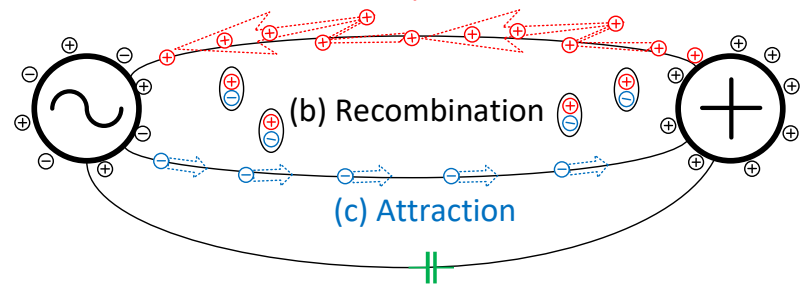

(d) Induction

Figure 6: Theoretical model of induction and ion current coupling

The corona pattern shows three bands of PD activity with random apparence and does not show any phase dependency. In contrast, the PRPD pattern shows a clear phase dependency, when an AC ripple is applied.

quantitative evaluation, the effect of relative ripple is displayed for the pulse amplitude (Figure $4 \mathrm{e}$ and repetition rate (Figure 4f). Both these parameters show an increase with relative ripple. The same is true for an increase in the applied DC voltage and surface gradient instead of

415 an $\mathrm{AC}$ ripple.

The corona activity is shown for DC energization in Figure $7 \mathrm{a}$, as well as for hybrid energization in Figure $7 \mathrm{~b}$. As no artificial sources are fixed on the AC conductor, no AC activity is visible in both figures. While some DC sources are already active for pure DC energization without coupling, they do not show the same degree of corona activity, as some sources are brighter than the others. In the case including the $\mathrm{AC}$ ripple, the number of active sources has clearly increased. Also, the DC discharges appear qualitatively brighter and longer. Furthermore, their activity seems more homogeneous.

\subsection{Discussion}

The DC corona has proven to be generally less stable than AC corona and shows more interference between adjacent corona sources, which is attributed to the effect of the space charge shielding adjacent sources as discussed in [9]. Therefore, also the corona UV example in Fig- ure $7 \mathrm{a}$ and Figure $7 \mathrm{~b}$ does not show a very homogeneous activity. This is also a possible explanation for the three bands of corona activity in the PRPD plot in Figure $7 \mathrm{c}$ and Figure $7 \mathrm{~d}$ As the different corona sources show different optical activity, it is logical that also the amplitude and repetition rate should be different. Another explanation would be the automatic range function of the PD sensor leading to concrete bands, however other PRPD plots for the same setup show discharges over the whole range, depending on applied voltage and $\mathrm{AC}$ ripple. Therefore, the authors are convinced, that the different bands of partial discharge are rather caused by the different state of corona on the single sources. This can also occur, as the u-type shape allows discharges to occur on different spots, with corona not always having the exact same onset conditions. textcolorredThe electrostatic in duction in Figure6(d) should cause an AC ripple on the DC conductor. As the worstcase is assumed to occur in dry weather with only DC corona, the other mechanisms based on the ion current coupling do not play a role in this example, but can of course also affect the DC corona in the case of rain, when the audible noise is lower. Based on the PRPD pattern in ${ }_{455}$ Figure $7 \mathrm{~d}$, the number of discharges as well as their amplitude is strongly reduced during the positive AC half-wave (equivalent to the negative half-wave of the ripple), as the surface gradient is reduced due to the lower potential difference between the $\mathrm{AC}$ and $\mathrm{DC}$ conductor. Thereby, the difference between the applied electric field and the critical electric field is increased in the negative half-wave, allowing the formation of longer streamers and allowing more pulses to occur. If the applied electric field is only slightly above the critical electric field, the ripple can push the corona further above the onset, resulting in a strong increase of the number of pulses, as seen in Figure $4 \mathrm{f}$ This trend can also be confirmed looking at the images of the corona activity in Figure $7 \mathrm{~b}$, where the activity of the DC corona sources appears more homogeneous, as the AC ripple pushes more sources above the offset. 


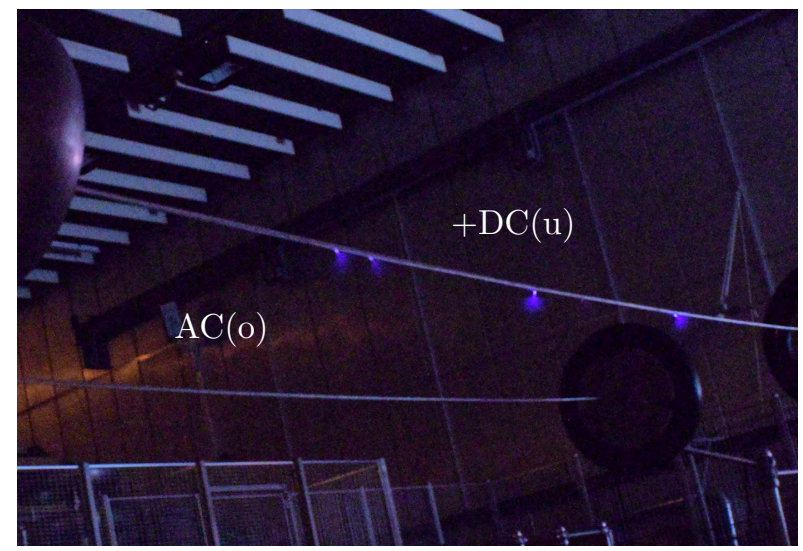

(a) DC corona activity for $0 \mathrm{kV}$ AC

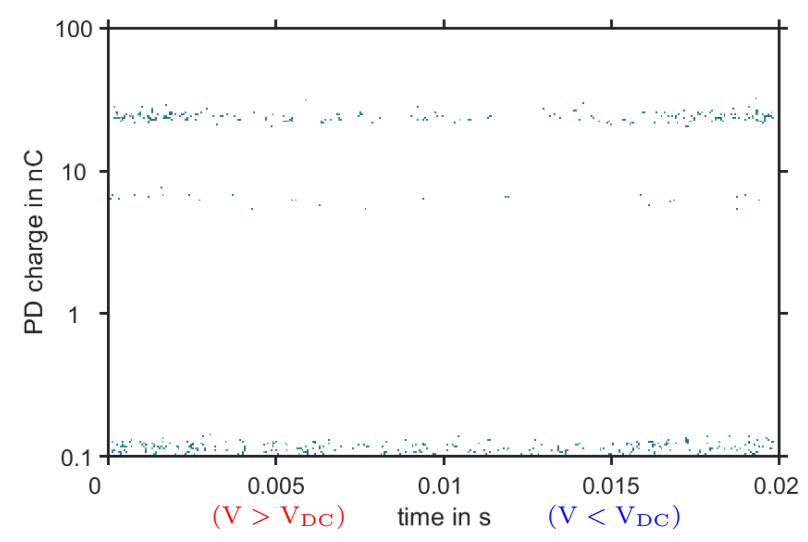

(c) DC Corona PRPD for the $0 \mathrm{kV} \mathrm{AC}$

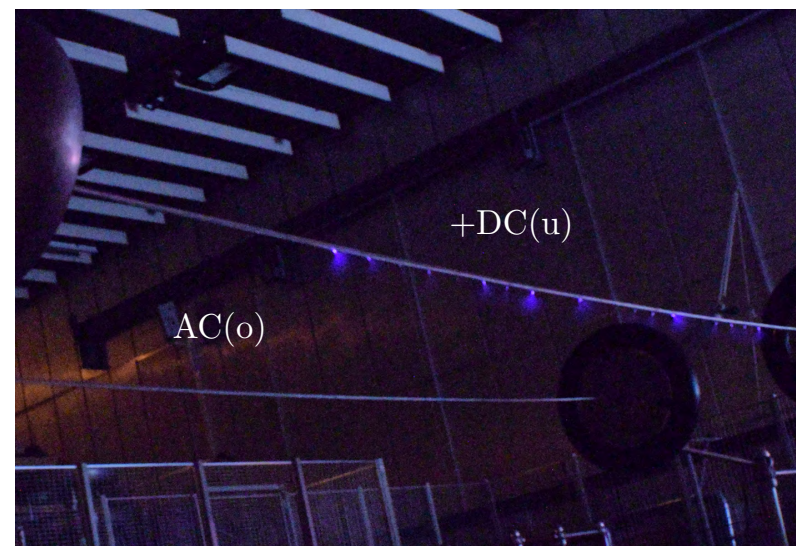

(b) DC corona activity for $150 \mathrm{kV} \mathrm{AC}$

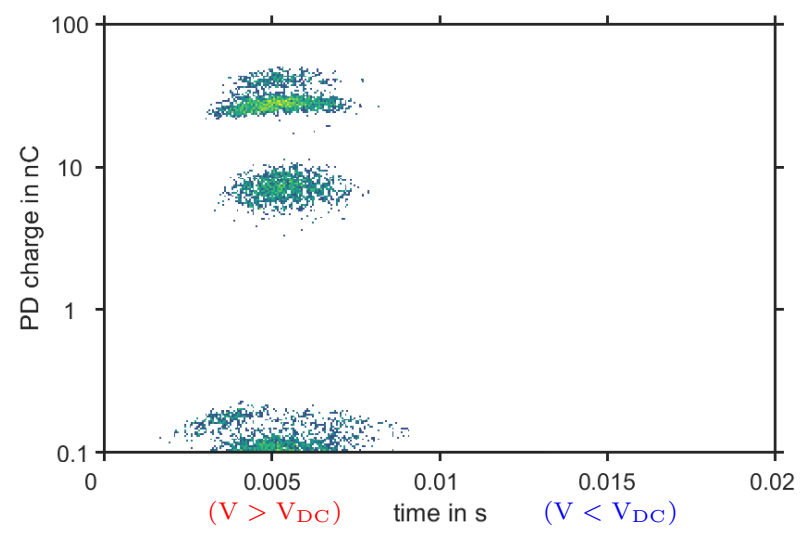

(d) DC Corona PRPD for $150 \mathrm{kV} \mathrm{AC}$

Figure 7: Comparison of DC corona PRPDs and UV activity at $180 \mathrm{kV}$ for no ripple and $150 \mathrm{kV} \mathrm{AC}$

\section{Conclusion \& outlook}

Based on a large series of small-scale experiments with typical overhead line conductors and realistic gradients and relative coupling, the $\mathrm{AC}$ and $\mathrm{DC}$ corona effects of hybrid AC/DC lines were investigated regarding DC bias or AC ripple caused by an adjacent bundle. It was demon-500 strated that the corona characteristics are effected by the relative coupling from the adjacent bundle. The PRPD patterns clearly show a change in corona activity in both halfwaves and allow to explain the observed trends in audible noise. Both, the repetition rate as well as the maximum pulse amplitudes are increased through relative coupling with respect to polarity. The optical investigation ${ }_{505}$ of the number and activity of the corona sources qualitatively confirm these results. In consequence of the altered corona activity, also the audible noise is affected. Therefore, the audible noise on a hybrid tower can be increased ${ }_{510}$ through a positive DC bias or AC ripple, but also possibly be reduced through a negative DC bias in some cases and therefore allows to design an optimized conductor arrangement for an improved corona performance. In order 515 to confirm these results for a full-scale overhead line under real weather conditions and pollution, a series of outdoor tests is scheduled on a recently constructed test line [24.
495 Acknowledgment

This work was financially supported by the National Research Program 'Energy Turnaround' (NRP 70) of the Swiss National Science Foundation (SNF) and the German TSO TransnetBW GmbH. The authors would like to thank Michiel Tavernier for the initial design of the corona sources, as well as Lorenz Bort, Pengfei Xu, Pascal Bleuer, Oliver Pischler and Antoine Gaillard for the fruitful discussions.

\section{References}

[1] S. Hedtke, M. Pfeiffer, C. M. Franck, C. Dermont, I. Stadelmann-Steffen, J. Jullier, HVDC \& hybrid HVAC/HVDC overhead line conversion: An acceptance case study, in: Cigre Session, 2018.

[2] CIGRE Working Group B2.41, Technical Brochure 583: Guide to the Conversion of Existing AC Lines to DC Operation, in: Cigré Technical Brochure 583 - Working Group B2.41, no. May, 2014.

[3] C. Neumann, B. Rusek, S. Steevens, K. H. Weck, Design and layout of AC-DC hybrid lines, in: Cigre Auckland Symposium, Auckland, 2013.

[4] W. Li, B. Zhang, R. Zeng, J. He, Study on the field effects under reduced-scale DC/AC hybrid transmission lines, IET

1. Generation, Transmission \& Distribution (7) 717-723. doi: 10.1049/iet-gtd.2012.0407 
[5] S. Hedtke, M. Pfeiffer, C. M. Franck, L. Zaffanella, J. Chan, J. Bell, Audible noise of hybrid AC / DC overhead lines : Comparison of different prediction methods and conductor arrangements, in: EPRI HVDC \& FACTS Conference, Palo-Alto, 2015.

6] P. S. Maruvada, Corona in Transmission Systems: Theory, De-595 sign and Performance, Eskom power series, Crown Publications.

[7] B. Clairmont, G. Johnson, L. Zaffanella, The effect of HVACHVDC line separation in a hybrid corridor, IEEE Transactions on Power Delivery (2) 1338-1350. doi:10.1109/61.25621

[8] U. Straumann, Berechnung und Reduktion der tonalen Geräus-600 chemission von Hochspannungsfreileitungen, Ph.D. thesis, ETH Zurich (2007). doi:10.3929/ethz-a-005486919

[9] L. D. Anzivino, G. Gela, W. W. Guidi, G. B. Johnson, J. J. LaForest, C. W. Nicholls, H. M. Schneider, L. E. Zaffanella, HVDC transmission line reference book, EPRI, 1993.

605

[10] M. Akazaki, Corona Phenomena from Water Drops on Smooth Conductors under High Direct Voltage, IEEE Transactions on

[ Power Apparatus and Systems 84 (1) (1965). doi:10.1109/ TPAS.1965.4766101

[11] F. Hirsch, E. Schafer, Progress Report on the HVDC Test Line610 of the $400 \mathrm{kV}$-Forschungsgemeinschaft: Corona Losses and Radio Interference, IEEE Transactions on Power Apparatus and Systems (7) 1061-1069. doi:10.1109/TPAS.1969.292506.

[12] L. E. Zaffanella, J. Doyl, F. J. Ellert, S. A. Miske, C. J. Truax, J. J. LaForest, T. S. Laube, M. G. Comber, D. W. Deno, Trans-615 mission Line Reference Book (1982) 625.

[13] V. L. Chartier, S. H. Sarkinen, R. D. Stearns, A. L. Burns, Investigation of Corona and Field Effects of AC/DC Hybrid Transmission Lines, IEEE Transactions on Power Apparatus and Systems (1) 72-80. doi:10.1109/TPAS.1981.316889

[14] B. Clairmont, L. E. Zaffanella, S. A. Sebo, D. G. Kasten, S. Zelingher, J. Hall, Hybrid Tower Study: Volume 3: Phase 3-Scale Model Development and Full-Scale Tests, EPRI.

[15] P. S. Maruvada, S. Drogi, Field and ion interactions of hybrid $\mathrm{AC} / \mathrm{DC}$ transmission lines, IEEE Transactions on Power Deliv-625 ery 3 (3) (1988) 1165-1172. doi:10.1109/61.193899

[16] M. Pfeiffer, S. Hedtke, C. M. Franck, Corona Current Coupling in Bipolar HVDC and Hybrid HVAC/HVDC Overhead Lines,

11 IEEE Transactions on Power Delivery (1) 393-402. doi:10. 1109/TPWRD . 2017.2713603

[17] S. Hedtke, M. Pfeiffer, A. Gaillard, C. M. Franck, Effect of electrostatic induction and space charges on the audible corona noise of hybrid AC/DC transmission lines, in: CIGRE-IEC Colloquium on EHV and UHV (AC \& DC), Montreal, 2016.

[18] Y. Liu, X. Cui, T. Lu, Z. Wang, X. Li, Y. Xiang, X. Wang, Detailed characteristics of intermittent current pulses due to positive corona, Physics of Plasmas (8). doi:10.1063/1.4892348.

[19] X. Li, J. Wang, Y. Li, Q. Zhang, T. Lu, X. Cui, Correlation between audible noise and corona current generated by $\mathrm{AC}$ corona discharge in time and frequency domains 063512 (2018). doi:10.1063/1.5020221

[20] N. G. Trinh, Particle Discharge XIX: Discharge in Air Part 1: Physical Mechanisms, Ieee Electrical Insulation Magazine (2) 23-29.

[21] J. M. Trüssel, H.-J. J. Weber, C. M. Franck, Safe and Automated Control of Experiments With a High Voltage Constuction Kit, The 19th International Symposium on High Voltage Engineering, Pilsen, Czech Republic, August, 23 - 28, 2015 (2015) $23-28$.

[22] F. Ianna, G. Wilson, D. Bosack, Spectral Characteristics of Acoustic Noise from Metallic Protrusions and Water Droplets in High Electric Fields, IEEE Transactions on Power Apparatus and Systems (6) 1787-1796. doi:10.1109/TPAS.1974.293830.

[23] P. S. Maruvada, R. D. Dallaire, P. Heroux, N. Rivest, Corona Studies for Biploar HVDC Transmission at Voltages Between \pm 600 kV AND \pm 1200 kV PART 2: Special Biploar Line, Bipolar Cage and Bus Studies, IEEE Transactions on Power Apparatus and Systems (3) 1462-1471. doi:10.1109/TPAS.1981.316621.

[24] S. Hedtke, M. Pfeiffer, M. Gobeli, P. Bleuler, R. Bräunlich, C. M. Franck, Setup of an outdoor hybrid AC / DC test line for corona measurements, in: VDE High Voltage Congress, 2018.

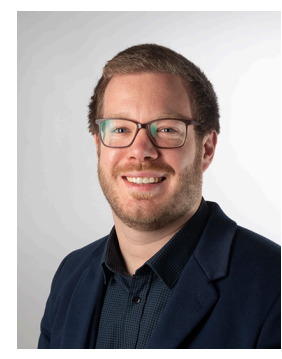

Sören Hedtke was born in 1988 in Neuwied, Germany and studied Electrical Engineering and Information Technology at TU Darmstadt (Germany) and the Grenoble INP (France). Since he received his M.Sc. degree in 2014, he is with the High Voltage Laboratory at ETH Zurich (Switzerland). His current Ph.D. research focuses on hybrid $\mathrm{AC} / \mathrm{DC}$ overhead lines with a special interest on the influence of coupling effects on corona phenomena and audible noise.

Martin Pfeiffer was born in Cotonou, Benin, in 1986. He received the M.Eng. degree in Engineering and Business Finance from University College London, UK, in 2008, the M.Sc. degree in Energy Science and Technology from ETH Zurich, Switzerland, in 2012 and the Ph.D. degree from ETH Zurich in 2017. His main research topics are corona effects on HVDC overhead lines and their impact on potential hybrid $\mathrm{AC} / \mathrm{DC}$ transmission lines.

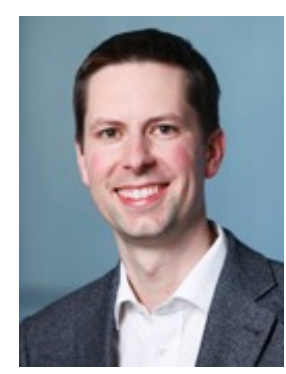

Christian M. Franck received a diploma in physics from the University of Kiel, Germany in 1999 and the Ph.D. degree in physics from the University of Greifswald, Germany in 2003. He was with the Swiss corporate research center of ABB from 2003-2009 as a Scientist and Group Leader for gas circuit breakers and high-voltage systems. Currently, he is Professor for High Voltage Technology at the Swiss Federal Institute of Technology (ETH) in Zurich, Switzerland. 